\title{
Using the Monogenic Signal for Cell-Background Classification in Bright-Field Microscope Images
}

\author{
Firas Mualla $^{1}$, Simon Schöll ${ }^{1,2,3}$, Björn Sommerfeldt ${ }^{4}$, Joachim Hornegger $^{1,3}$ \\ ${ }^{1}$ Pattern Recognition Lab, Friedrich-Alexander University Erlangen-Nuremberg \\ ${ }^{2}$ ASTRUM IT GmbH, Erlangen \\ ${ }^{3}$ SAOT Graduate School in Advanced Optical Technologies \\ ${ }^{4}$ Institute of Bioprocess Engineering, Friedrich-Alexander University \\ Erlangen-Nuremberg \\ firas.mualla@cs.fau.de
}

\begin{abstract}
Some cell detection approaches which deal with bright-field microscope images utilize defocussing to increase the image contrast. The latter is related to the physical light phase through the transport of intensity equation (TIE). Recently, it was shown that it is possible to approximate the solution of the TIE using a modified monogenic signal framework. We show empirically that using the local phase of the previous monogenic signal in place of the defocused image improves the cellbackground classification rate. The evaluation was performed on L929 adherent cell line with more than 1000 manually labeled cells. The improvement was $6.8 \%$ using a random forest classifier and $10 \%$ using a support vector machine classifier with a radial basis function kernel.
\end{abstract}

\section{Introduction}

Detecting cells in microscope images is a crucial step in the cell image analysis. Several approaches in different image modalities tackle the problem as a classification problem. A fixed-size square patch is sampled at each pixel and used to train a cell-background classifier. The features can be either the patches themselves as in [1] or the patches after applying traditional feature extraction schemes as in [2],[3],[4].

It is known that bright-field microscopy delivers insufficient contrast at focus especially for the adherent cells [5],[6]. More contrast can be obtained by defocussing the microscope [5]. Moreover, in quantitative phase microscopy (QPM) approaches, the physical light phase can be reconstructed computationally from the amplitude information in order to get both more contrast and more object details.

A QPM approach in [7] suggests approximating the TIE (section 2.1) solution in the monogenic signal (section 2.2) domain. In fact, the obtained results approximate the local phase and the local energy of the physical light phase.

It is expected that the defocused image delivers higher discrimination between the background and the cells compared to the at-focus image. In this paper, we show that using the previously mentioned local phase instead of the 
defocused image yields even higher discrimination power for the cell-background classification problem. Section 2.3 discusses the details of the used classifier models and features.

The experiments were performed on bright-field images of an unstained adherent L929 cell culture. Section 2.4 clarifies the acquisition and the labeling details. Section 3 shows the results of these experiments which are further discussed and summarized in section 4 .

\section{Materials and Methods}

\subsection{Transport of intensity equation}

As mentioned in the introduction, defocussing a bright-field microscope yields more contrast in the acquired images. In fact, there is a relation between this contrast and the physical phase of light. The transport of intensity equation TIE [8] models this relation:

$$
\frac{2 \pi}{\lambda} \frac{\partial I}{\partial z}=-\nabla \cdot I \nabla \phi
$$

Where $\lambda$ is the wavelength of light, $I$ is the intensity image at the defocus distance $z$, and $\phi$ is the physical phase of light.

\subsection{Monogenic signal}

The monogenic signal is a 2D generalization of the analytic signal [9]. Like its 1D counterpart, it is computed in practice by convolving the signal with a band-pass quadrature filter yielding the local phase and local energy of the input.

In [7], a link between the physical phase and the local phase was established using the monogenic signal. According to [7], it is possible to use the monogenic signal framework to approximate the solution of equation (1) under two conditions: First, the derivative image, i.e. the left side of equation (1), is used as an input instead of the image itself. Second, a low-pass filter is used in the monogenic signal framework instead of the band-pass filter.

\section{$2.3 \quad$ Learning}

We want to investigate the discriminative power of the local phase as defined in section 2.2 compared to the defocused images in the cell-background separation problem. Obviously, it is possible to measure the discriminative power difference by learning a classifier for each of them and then comparing the test errors.

As a classifier model, we use the support vector machine (SVM) and the random forest $(\mathrm{RF})$. The kernel of the SVM was set to the radial basis function (RBF) kernel. The cost parameter and the RBF $\gamma$ parameter were set to the default parameters in LibSVM. The trees number in the RF and the number of the randomly selected variables at each node were set following [10] to 500 and $N / 5$, respectively. $N$ is the feature number. 
The feature vectors are $5 X 5$ patches. Therefore, the number of features is 25 . We did not conduct a thorough analysis of the effect of the patch size. However, cell areas in our data are considerably larger than the chosen patch area.

The data was z-scored for the SVM, while it was used without normalization for the RF.

Cutting patches at each pixel is computationally expensive. Therefore, only $P$ patches are randomly sampled from each image. $P$ was set to 100 .

\subsection{Materials}

The evaluation was performed on L929 adherent cells. The images were acquired with an inverted Nikon Eclipse TE2000U microscope using Nikon USB camera. The used microscope objective has a numerical aperture of 0.45 , a working distance of $7.4 \mathrm{~mm}$, and 20x magnification. Image resolution is $1280 \mathrm{X} 960$ pixels with $0.49 \mu \mathrm{m} /$ pixel.

The acquired data consists of five pairs of images. Each pair consists of an image at focus (figure 1(a)) and another positively defocused image (figure 1(b)) of the same scene at distance $+30 \mu \mathrm{m}$. The total number of cells is 1078. All of them were labeled by two bioprocess engineering experts. This was done by manually delineating the borders of the cells in the defocused images.

The software SePhaCe [6] was used to generate the local phase (figure 1(c)) and the local energy (figure 1(d)) images for each image pair.

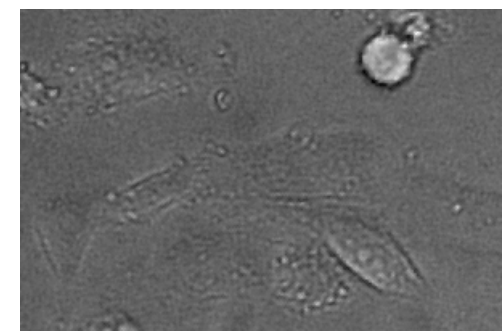

(a) At-focus

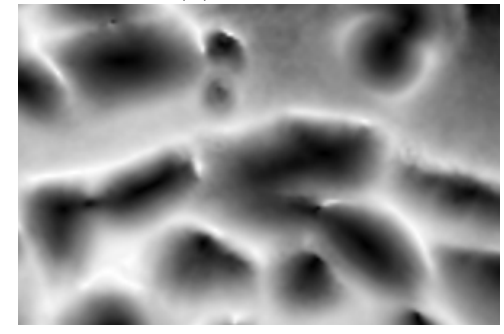

(c) Local phase

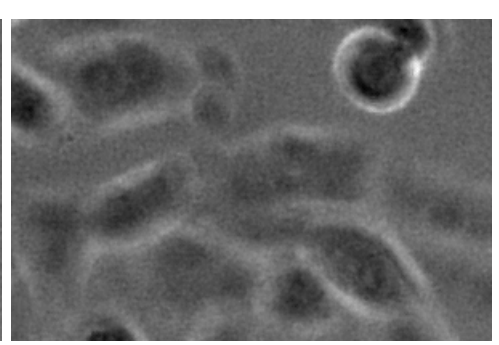

(b) Defocused

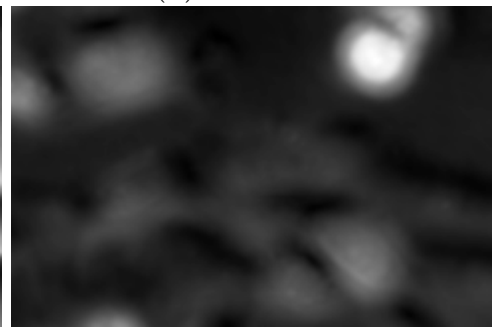

(d) Local energy

Fig. 1. Examples cut from evaluation images. The histograms of all four cuts were linearly stretched for the clarity. 
Mualla et al.

\section{Results}

One of the five at-focus images was used to train both the SVM and the RF, then the learned models were applied on the other at-focus images. This was repeated for each at-focus image and the mean test error was computed. The previous experiment was repeated 10 times with one mean test error obtained from each repetition. The mean and the standard deviation of all these mean test errors are shown in the first column of table 1.

The same was done on the positively defocused images and the results are shown in the second column of the table.

The third and the fourth columns show the results when the same process applied on the local phase and the local energy, respectively.

The results show that the defocused image contains more discriminative power than the at-focus image, while the local phase contains more discriminative power than the defocused image.

\begin{tabular}{|c|c|c|c|c|}
\hline & at-focus & defocused & phase & energy \\
\hline RBF SVM & $39.9 \% \pm 3.6 \%$ & $32.8 \% \pm 2.5 \%$ & $\mathbf{2 2 . 8} \% \pm \mathbf{1 . 7} \%$ & $33.6 \% \pm 3.4 \%$ \\
\hline RF & $45.0 \% \pm 3.3 \%$ & $32.0 \% \pm 2.6 \%$ & $\mathbf{2 5 . 2} \% \pm \mathbf{1 . 1} \%$ & $37.6 \% \pm 2.4 \%$ \\
\hline
\end{tabular}

Table 1. Comparing the discriminative power of the at-focus, the defocused, and the monogenic output using classification test errors.

\section{Conclusion and discussion}

We have empirically showed that the pixelwise cell-background classification yields considerably better results when the local phase as obtained in [7] is used instead of the defocused image. Nevertheless, the defocused image still delivers better results compared to the at-focus image.

More than 1000 manually labeled adherent cells were used in the evaluation. This relatively large number of cells supports the soundness of the paper statement.

One might criticize the evaluation as being done using one defocus distance, i.e. the distance of $30 \mu \mathrm{m}$ described in section 2.4. Actually, the very short distances do not deliver sufficient contrast. On the other hand, very long distances smash out the image information due to the excessive blurring by the point spread function of the optical system. Therefore, there is an optimal distance which maximizes the contrast. During the image acquisition, we tried to pick out this optimal distance experimentally. However, this was judged subjectively. We are currently developing methods to choose this distance objectively. In fact, other factors should be considered for the defocus distance selection because it is used to estimate a derivative image. Further research will tackle this issue. 


\section{Acknowledgment}

The authors would like to thank the Bavarian Research Foundation BFS for funding the project COSIR under contract number AZ-917-10.

\section{References}

1. Jesper Sjöström P, Frydel BR, Wahlberg LU. Artificial neural network-aided image analysis system for cell counting. Cytometry. 1999;36(1):18-26.

2. Nattkemper TW, Ritter H, Schubert W. Extracting patterns of lymphocyte fluorescence from digital microscope images. Intelligent Data Analysis in Medicine and Pharmacology. 1999;99:79-88.

3. Long X, Cleveland WL, Yao YL. A new preprocessing approach for cell recognition. IEEE Transactions on Information Technology in Biomedicine. 2005;9(3):407-412.

4. Long X, Cleveland WL, Yao YL. Automatic detection of unstained viable cells in bright field images using a support vector machine with an improved training procedure. Computers in Biology and Medicine. 2006;36(4):339-362.

5. Agero U, Monken CH, Ropert C, Gazzinelli RT, Mesquita ON. Cell surface fluctuations studied with defocusing microscopy. Physical Review E. 2003;67(5):051904.

6. Ali R, Gooding M, Szilágyi T, Vojnovic B, Christlieb M, Brady M. Automatic segmentation of adherent biological cell boundaries and nuclei from brightfield microscopy images. Machine Vision and Applications. 2012;23(4):607-621.

7. Ali R, Szilagyi T, Gooding M, Christlieb M, Brady M. On the Use of Low-Pass Filters for Image Processing with Inverse Laplacian Models. Journal of Mathematical Imaging and Vision. 2010; p. 1-10.

8. Teague MR. Deterministic phase retrieval: a Green's function solution. Journal of the Optical Society of America. 1983;73(11):1434-1441.

9. Felsberg M, Sommer G. The monogenic signal. IEEE Transactions on Signal Processing. 2001;49(12):3136-3144.

10. Khoshgoftaar TM, Golawala M, Van Hulse J. An empirical study of learning from imbalanced data using random forest. In: Proceedings of the IEEE International Conference on Tools with Artificial Intelligence. 2; 2007. p. 310-317. 